\title{
Effect of T'ai Chi Exercise on Hypertension in Young and Middle-Aged In-Service Staff
}

\author{
Xiao-Ling Shou, MS, Lei Wang, MS, Xiao-Qing Jin, MD, Li-Yue Zhu, MS, \\ Ai-Hua Ren, MB, and Qi-Nan Wang, $M^{4}{ }^{4}$
}

\begin{abstract}
Objective: This study aims to investigate the effect of t'ai chi exercise on hypertension in young and middleaged in-service staff.

Methods: A total of 208 subjects with grade 1 hypertension were enrolled into this study. These subjects were randomly divided into two groups: research group and control group $(n=104$, each). On the basis of general daily lifestyle intervention, subjects in the research group underwent 24-Style Simplified t'ai chi exercise for 3 months, whereas subjects in the control group underwent general daily lifestyle intervention. All subjects were followed up at the first and third month of intervention. The body mass index (BMI), blood pressure, blood lipid, and other indexes were measured before and after the intervention, and quality of life was evaluated.

Results: (1) In the research group, after 1 month of exercise, systolic blood pressure (SBP), heart rate (HR), triglyceride (TG), total cholesterol (TC), and low-density lipoprotein cholesterol (LDL-C) significantly decreased $(p<0.05)$, while BMI, blood glucose (Glu), diastolic blood pressure (DBP), and pulse pressure (PP) did not significantly change. Furthermore, after 3 months of exercise, BMI, HR, SBP, DBP, PP, TG, TC, LDL-C, and Glu all significantly decreased $(p<0.05)$. (2) Moreover, the quality of life of subjects in the research group obviously improved after 3 months of $t$ 'ai chi exercise $(p<0.05)$.

Conclusions: $t$ 'ai chi exercise can reduce the level of blood pressure in young and middle-aged in-service staff with grade 1 hypertension, control weight, slow down the HR, improve metabolism, and improve quality of life. t'ai chi is an exercise suitable for in-service hypertension subjects.
\end{abstract}

Keywords: $t$ 'ai chi exercise, hypertension, the cardiovascular system, the quality of life

\section{Introduction}

$\mathbf{H}$ YPERTENSION IS ONE of the common diseases that threaten human health, and is the main risk factor for stroke, coronary heart disease, and death. ${ }^{1,2}$ An investigation revealed that the prevalence rate of hypertension in college and university faculty in Zhejiang was $17.2 \%$, and the prevalence of prehypertension was $40 \%$, which is higher than the prevalence of prehypertension at the age of 18-39 years $(37.8 \%)$, as revealed by the "China Health and Nutrition Survey" carried out within 1991-2009. ${ }^{3}$ Obesity, overweight, and smoking are the main factors that affect blood pressure. Exercise has gradually shown an irreplaceable role in the hypertension population, especially in the process of prevention, treatment, control, and rehabilitation of hypertension. Literatures have revealed that exercise can effectively improve the condition of blood pressure, and the occurrence and development of hypertension are greatly correlated with exercise. Continuous aerobic exercise can not only reduce the side effects of drugs but also reduce medical costs for subjects with hypertension. ${ }^{4}$ t'ai chi is one of the traditional fitness sports in China. t'ai chi stresses relaxed, natural, spiritual, supple, and coherent actions, and edifying sentiment and improving self-cultivation. Hence, it continues to be popular in more and more people. ${ }^{5}$ A study has reported the effect of $t$ 'ai chi on the long-term quality of life of elderly subjects with hypertension. ${ }^{6}$ However, at present, there is no report on the detailed role of t'ai chi in in-service

Departments of ${ }^{1}$ Cardiac Rehabilitation, ${ }^{2}$ Mental Health, ${ }^{3}$ Traditional Chinese Medicine, and ${ }^{4}$ Rehabilitation Center, Zhejiang Hospital, Hangzhou, China. 
hypertension subjects at home and abroad. This study was based on strict medical research principles. Starting with the screening of the subjects, personalized intervention plans were proposed for the assessment of the patient's physical condition through a baseline test. Then, the active influence of t'ai chi on in-service hypertension subjects was further understood through observation. This provides a new idea and basis for the prevention and treatment of hypertension in the in-service population.

\section{Subjects and Methods}

\section{Subjects}

A total of 208 subjects with grade 1 hypertension were enrolled into this study. These subjects were randomly divided into two groups: research group and control group ( $n=104$, each). Five subjects were lost and five subjects withdrew during the trial. Finally, 98 subjects in the research group and 100 subjects in the control group completed the trial. In the research group, 48 subjects were male (average age: $52 \pm 6.46$ years) and 50 subjects were female (average age: $51 \pm 7.09$ years). Furthermore, among the subjects in the research group, 42 subjects were at low risk, whereas 56 subjects were at moderate risk. In the control group, 55 subjects were male (average age: $52 \pm 8.98$ years) and 45 subjects were female (average age: $51 \pm 7.54$ years). Furthermore, among the subjects in the control group, 46 subjects were at low risk, whereas 54 subjects were at moderate risk.

Inclusion criteria. All candidates met the diagnostic criteria for primary grade 1 hypertension in the Guidelines for Prevention and Treatment of Hypertension in China (2010 revised version): subjects with a systolic blood pressure (SBP) of $\geq 140 \mathrm{mmHg}$ and $<160 \mathrm{mmHg}$ and/or a diastolic blood pressure (DBP) of $\geq 90 \mathrm{mmHg}$ and $<100 \mathrm{mmHg}$, subjects without risk factors and the target organ damage are defined as low risk, and subjects with one to two risk factors defined as moderate risk. ${ }^{7}$ The age of these subjects ranged within 18-60 years. These subjects were in-service, had low mobility and good compliance, could accept t'ai chi exercise, would not change their post, and would not withdraw from the trial within half a year. Furthermore, the subjects were newly diagnosed subjects who did not take antihypertensive or lipid-lowering drugs.

Exclusion criteria. Subjects who had secondary hypertension, coronary heart disease, diabetes, or stroke; subjects who were pregnant or lactating, or were fitness/sports coaches; subjects who could not provide a signed informed consent due to mental illness; subjects who had physical activity disorder; subjects who could not well cooperate due to their busy work schedule were excluded. During the period of the study, when their blood pressure continued to rise to the standard of primary grade 2 hypertension or serious adverse events occurred, the patient was given standardized drug treatment, and withdrew from the study.

\section{Methods}

Control group. Subjects in the control group were given general daily lifestyle intervention (hypertension knowledge propaganda, propaganda for blood pressure monitoring, and healthy lifestyle self-management, such as persuasion for smoking cessation, alcohol restriction, sodium restriction, dietary balance, weight control, and general daily exercise).

Research group. On the basis of general daily lifestyle intervention, subjects in the research group underwent the 24-Style Simplified t'ai chi exercise for 3 months. Before the trial, subjects in the research group underwent 2 weeks of learning the 24-Style Simplified t'ai chi. These subjects were directed by professional rehabilitative therapists to ensure that all subjects were proficient in t'ai chi exercise. Practice was in strict accordance with the requirements of t'ai chi training, and their movements and postures, styles and intension were all stressed.

Choice of exercise intensity. The target heart rate (HR) during the exercise was set as $50 \%-60 \%$ of the maximal oxygen uptake or $70 \%-80 \%$ of the maximum $\mathrm{HR}\left(\mathrm{HR}_{\max }\right)$, which was calculated using the formula: target $\mathrm{HR}=$ resting $\mathrm{HR}+$ resting $\mathrm{HR} \times(50 \%-70 \%)$. The duration of each exercise was $40-90 \mathrm{~min}$. Preparatory activities were first performed before each exercise, which comprised of 10-15 min of walking or conditioning exercise. Then, the 24-Style t'ai chi was completed (repeated two to four times, for 20$30 \mathrm{~min}$ ). The exercise intensity was best when the target HR was achieved in 20-30 min, and the HR returned to normal levels at 5-10 min after the exercise. Exercise intensity was appropriate when the patient did not feel out of breath, uncomfortable, and dizzy. The exercise was continued when the participant felt relaxed, energetic, and conscious, subjective symptoms were improved, and their blood pressure was down and stable after every exercise. When opposite situations occurred, the exercise was stopped, and retraining was performed when the situation improved. According to the exercise time that most residents used, the exercise time was arranged in a fixed leisure time in the morning and evening. The exercise was completed one to two times a day. 8,9

Blood pressure measurement. A mercury sphygmomanometer was used to measure blood pressure. The staff who participated in the blood pressure measurement were trained by a unified standard based on the Chinese Guidelines for Blood Pressure Measurement 2010, ${ }^{7}$ and passed the qualification test for blood pressure measurement. Before the measurement, the patient sat and remained at rest for $5 \mathrm{~min}$. Blood pressure was measured three times using the Korotkoff method, with intervals longer than $1 \mathrm{~min}$. Then, the average value was calculated.

Measurement of the physical index. The measurement was carried out according to the requirements in "The Manual of Physical Fitness Monitoring for Chinese Adults." The height and body weight were measured using a Tsinghua Tongfang National Physical Tester.

Detection of biochemical indexes. The biochemical indexes of subjects in these two groups were detected before the trial, and at 1 month and 3 months during the trial. After $12 \mathrm{~h}$ of fasting, $5 \mathrm{~mL}$ of fasting venous blood was collected, the sample was left standing, centrifuged at 4,000 rpm for $10 \mathrm{~min}$, and the serum was used to detect blood lipid and other indexes using a biochemistry analyzer. 
Table 1. Changes in Blood Pressure and Body Mass Index

\begin{tabular}{lllll}
\hline Groups & \multicolumn{1}{c}{$S B P(\mathrm{mmHg})$} & $D B P(\mathrm{mmHg})$ & $P P(\mathrm{mmHg})$ & $B M I\left(\mathrm{~kg} / \mathrm{m}^{2}\right)$ \\
\hline Control group & & & & \\
Baseline & $142.90 \pm 7.91$ & $82.40 \pm 7.82$ & $59.79 \pm 8.23$ & $26.71 \pm 1.90$ \\
At the end of the first month & $143.10 \pm 7.92$ & $81.39 \pm 5.42$ & $60.90 \pm 8.21$ & $26.68 \pm 1.87$ \\
At the end of the third month & $142.91 \pm 6.80$ & $83.29 \pm 7.80$ & $59.10 \pm 7.34$ & $26.67 \pm 1.90$ \\
Research group & & & & \\
$\quad$ Baseline & $139.42 \pm 9.47$ & $83.20 \pm 9.45$ & $55.23 \pm 11.44$ & $26.71 \pm 1.70$ \\
At the end of the first month & $132.45 \pm 10.29^{* * * *}$ & $79.91 \pm 6.20$ & $53.07 \pm 9.24^{*}$ & $26.43 \pm 3.10$ \\
At the end of the third month & $126.68 \pm 9.87^{* * * *}$ & $76.28 \pm 7.79^{* * * *}$ & $50.40 \pm 10.44^{* * * *}$ & $25.83 \pm 2.71^{* *}$ \\
\hline
\end{tabular}

Intergroup $t$ test: all compared with the control group, ${ }^{*} p<0.05$; intragroup $t$ test: all compared with the baseline, $* * p<0.05$. BMI, body mass index; DBP, diastolic blood pressure; PP, pulse pressure; SBP, systolic blood pressure.

Assessment of quality of life. The quality of life of subjects in these two groups was evaluated using the Short Form Health Survey (SF-36QOL). The higher the score was, the better the quality of life is. ${ }^{10}$

\section{Statistical analysis}

All data were processed using statistical software SPSS 16.0. Measurement data were expressed as mean \pm standard error of measurement $(x \pm S E M)$. The comparison of variables before and after the experiment in the same group was conducted using paired $t$ test. Comparisons of variables before and after the experiment between the two groups were conducted using independent samples $t$ test. A value of $p<0.05$ was considered statistically significant.

\section{Results}

(1) Changes in blood pressure, pulse pressure (PP), and body mass index (BMI, Table 1)

\section{SBP results}

In the research group, the SBP before exercise was not significantly different than that in the control group. However, at the end of the first and third month of exercise, SBP greatly decreased compared with that at baseline level, and the difference was statistically significant $(p<0.05)$.

\section{$D B P$ results}

In the research group, the difference in DBP among the baseline level, the level at the end of the first month, and the level in the control group was not statistically significant. However, at the end of the third month, DBP statistically decreased $(p<0.05)$. In the research group, DBP did not significantly change at the end of the first month of exercise, when compared with that at baseline level. At the end of the third month, DBP significantly decreased $(p<0.05)$.

\section{PP results}

The difference in PP between the baseline level in the research group and the level in the control group was not statistically significant. At the end of the first and third month of exercise, PP significantly decreased $(p<0.05)$. In the research group, PP did not significantly change at the end of the first month of exercise, compared with the baseline level. However, at the end of the third month, PP greatly decreased $(p<0.05)$. In the control group, SBP, DBP, and PP did not significantly change, when compared with the baseline level.

\section{$B M I$ results}

The difference in BMI between the research group and control group was not statistically significant. In the research group, the difference in BMI at the end of the first month of exercise and at the baseline level was not statistically significant, while BMI greatly decreased at the end of the third month of exercise, compared with that at baseline level $(p<0.05)$. In the control group, BMI did not significantly change.

(2) At the end of the first and third month of exercise, the immediate blood pressure of subjects with hypertension significantly improved, and both immediate SBP and DBP significantly decreased $(p<0.01$ and $p<0.05$, respectively). Under the intervention of $t$ 'ai chi exercise, the immediate HR of the patient also significantly decreased, especially at the third month of exercise, and the decrease was more significant $(p<0.01$, Table 2).

(3) Changes in the metabolism of blood lipids: Differences in the level of total cholesterol (TC), triglyc-

Table 2. Changes in Instant Blood Pressure and Heart Rate of Patients in the Research Group (X \pm Standard Deviation)

\begin{tabular}{lrrr}
\hline Items & Baseline & At the end of the first month & At the end of the third month \\
\hline Instant systolic pressure $(\mathrm{mmHg})$ & $151.43 \pm 7.40$ & $146.40 \pm 5.24^{* *}$ & $142.10 \pm 5.41^{* *}$ \\
Instant diastolic pressure $(\mathrm{mmHg})$ & $84.59 \pm 8.10$ & $83.10 \pm 5.80^{*}$ & $81.73 \pm 5.51^{*}$ \\
Instant heart rate (times/min) & $102.70 \pm 8.40$ & $96.70 \pm 8.20^{*}$ & $95.10 \pm 6.40^{* *}$ \\
\hline
\end{tabular}

Intergroup $t$ test: all compared with the baseline, ${ }^{*} p<0.05$, $* * p<0.01$. 
Table 3. Changes in the Metabolism of Blood Lipids

\begin{tabular}{|c|c|c|c|c|}
\hline Groups & $T C(\mathrm{mmol} / \mathrm{L})$ & $T G(\mathrm{mmol} / \mathrm{L})$ & $H D L-C(\mathrm{mmol} / \mathrm{L})$ & $L D L-C(\mathrm{mmol} / \mathrm{L})$ \\
\hline \multicolumn{5}{|l|}{ Control group } \\
\hline Baseline & $5.67 \pm 0.96$ & $2.04 \pm 1.40$ & $1.40 \pm 0.40$ & $3.25 \pm 0.83$ \\
\hline At the end of the first month & $5.38 \pm 1.17$ & $1.94 \pm 0.76$ & $1.31 \pm 0.41$ & $2.99 \pm 0.97$ \\
\hline At the end of the third month & $5.69 \pm 1.11$ & $2.17 \pm 0.67$ & $1.29 \pm 0.42$ & $3.26 \pm 0.94$ \\
\hline \multicolumn{5}{|l|}{ Research group } \\
\hline Baseline & $5.79 \pm 0.91$ & $2.00 \pm 1.70$ & $1.49 \pm 0.26$ & $3.13 \pm 0.81$ \\
\hline At the end of the first month & $5.16 \pm 0.86^{*}$ & $1.40 \pm 0.58 *$ & $1.48 \pm 0.29$ & $2.49 \pm 0.77 *$ \\
\hline At the end of the third month & $5.20 \pm 0.79 *$ & $1.38 \pm 0.46^{*}$ & $1.49 \pm 0.25$ & $2.60 \pm 0.49 *$ \\
\hline
\end{tabular}

Intragroup $t$ test: compared with the baseline, ${ }^{*} p<0.05$.

TC, total cholesterol; TG, triglyceride; HDL-C, high-density lipoprotein cholesterol; LDL-C, low-density lipoprotein cholesterol.

eride (TG), high-density lipoprotein cholesterol, and low-density lipoprotein cholesterol (LDL-C) between the baseline levels of the research group and control group were not statistically significant. Furthermore, the levels of TC, TG, and LDL-C significantly decreased at the end of the first and third month of exercise, when compared with baseline levels $(p<0.05)$. In the control group, the levels of TC, TG, and LDL-C did not significantly change (Table 3 ).

(4) Results of measurement of quality of life: In the research group, the difference in quality of life before and after the intervention was statistically significant $(p<0.05)$. In the control group, the difference in quality of life before and after the intervention was not statistically significant ( $p>0.05$, Table 4$)$.

\section{Discussion}

Hypertension is the most important risk factor for cardiocerebrovascular diseases in China, and is the main cause of death of cardiocerebrovascular diseases in China. Controlling hypertension is the breakthrough point and key measure for the prevention of cardiocerebrovascular diseases. ${ }^{11}$ As a social population, in-service staff have many comprehensive factors, such as heavy tasks, mental stress, great psychological pressure, lack of exercise, and neglecting health care, which lead to elevated blood pressure, making them a hypertension-susceptible population, thereby becoming one of the high-risk populations of cardiovascular diseases.

At present, many scholars have revealed that small- and medium-intensity aerobic exercises have significant anti- hypertensive effects. Aerobic exercises, such as t'ai chi, walking, square dance, and yoga, can produce good effects on subjects with primary hypertension. ${ }^{12-16}$ A meta-analysis revealed that ${ }^{14}$ aerobic exercise could reduce the resting SBP and DBP of hypertensive subjects by 8 and $5 \mathrm{mmHg}$, respectively. Lund Rasmussen et al. revealed in a study that $^{15}$ a single time of aerobic exercise could reduce 24-h $\mathrm{SBP}$ by $2.4 \mathrm{mmHg}$. Furthermore, Dimeo et al. revealed in a study on the intervention of refractory hypertension that ${ }^{16}$ after 3 months of aerobic exercise, SBP and DBP decreased by an average of 6 and $3 \mathrm{mmHg}$, respectively.

This study suggests that t'ai chi exercise can improve the SBP, DBP, and PP of subjects to a certain extent. Lee revealed through a 6-month study that ${ }^{17}$ t'ai chi exercise could improve the waistline and blood pressure of elderly subjects. Eom $^{18}$ conducted a 12-month $t^{\prime}$ ai chi study that involved middle-aged women, and the results revealed that $t$ 'ai chi exercise could improve the risk factors of metabolic syndrome such as DBP and waistline. These were consistent with the results of this study. As an aerobic exercise, $t$ 'ai chi can effectively reduce the level of blood pressure in inservice staff with hypertension. ${ }^{19}$

This study revealed that t'ai chi exercise could improve the metabolism of lipid in subjects, and reduce the levels of TC, TG, and LDL-C. Furthermore, previous studies revealed that t'ai chi, as a low-intensity aerobic exercise, could open the reserved capillaries in the body, improve the metabolism of the body, improve the activity of enzymes to a certain extent, improve the metabolism of fat, accelerate the decomposition of low-density lipoprotein, and remove the inhibition of high-density lipoprotein. ${ }^{20,21}$ The disturbance of blood lipid metabolism is a risk factor in subjects with

Table 4. Comparison of SF-36 Score Before and After Intervention (Points, X \pm Standard Deviation)

\begin{tabular}{|c|c|c|c|c|c|c|c|c|}
\hline Groups & $\begin{array}{l}\text { Physiological } \\
\text { function }\end{array}$ & $\begin{array}{c}\text { Social } \\
\text { function }\end{array}$ & $\begin{array}{l}\text { Social } \\
\text { role }\end{array}$ & $\begin{array}{l}\text { Emotional } \\
\text { role }\end{array}$ & $\begin{array}{l}\text { Mental } \\
\text { health }\end{array}$ & Vitality & $\begin{array}{l}\text { Bodily } \\
\text { pain }\end{array}$ & $\begin{array}{l}\text { Total } \\
\text { health }\end{array}$ \\
\hline \multicolumn{9}{|l|}{ Control group } \\
\hline Baseline & $70.5 \pm 8.1$ & $60.2 \pm 6.3$ & $57.7 \pm 7.1$ & $60.1 \pm 7.4$ & $62.3 \pm 5.9$ & $60.1 \pm 6.1$ & $62.1 \pm 7.9$ & $61.8 \pm 9.6$ \\
\hline $\begin{array}{l}\text { At the end of the } \\
\text { third month }\end{array}$ & $75.3 \pm 8.7$ & $60.9 \pm 8.3$ & $58.2 \pm 15.4$ & $61.1 \pm 16.1$ & $63.6 \pm 13.41$ & $58.3 \pm 12.4$ & $66.9 \pm 13.8$ & $64.9 \pm 11.9$ \\
\hline \multicolumn{9}{|l|}{ Research group } \\
\hline Baseline & $71.1 \pm 8.8$ & $60.6 \pm 6.1$ & $58.1 \pm 5.9$ & $60.3 \pm 7.7$ & $61.2 \pm 6.6$ & $60.4 \pm 5.9$ & $63.9 \pm 7.7$ & $61.1 \pm 9.3$ \\
\hline $\begin{array}{l}\text { At the end of the } \\
\text { third month }\end{array}$ & $83.4 \pm 6.6^{*}$ & $80.1 \pm 19.3 *$ & $69.7 \pm 17.3^{*}$ & $76.5 \pm 17.9^{*}$ & $77.1 \pm 15.9^{*}$ & $69.1 \pm 11.9 *$ & $79.1 \pm 16.8^{*}$ & $77.6 \pm 16.3^{*}$ \\
\hline
\end{tabular}

Intragroup $t$ test: comparison before and after the research group intervention. ${ }^{*} p<0.05$. 
hypertension. In this study, 3 months of t'ai chi exercise was conducive to optimizing the body composition of subjects with hypertension, reducing body fat rate, improving the serum levels of TC, TG, and LDL-C, inducing a good regulating effect on the disorder of lipid metabolism, and reducing the risk factors of hypertension.

Health-related quality of life is an important indicator of health status, and the SF-36 is a set of generic, coherent, and easily administered quality-of-life measures. These measures rely upon patient self-reporting, and are now widely utilized by managed care organizations and by Medicare for routine monitoring and assessment of care outcomes in adult patients. Heckbert et al. revealed in a study that ${ }^{22}$ anxiety could induce the patient in a tension state, increase vascular tonicity, contract the arteriole, increase peripheral resistance, and accordingly increase blood pressure. Emotional problems can also induce the disturbance of regulation of the function of plant nerves and the function of the hypothalamus-pituitary gland-adrenaline axis, causing a highlevel state of blood pressure in subjects. ${ }^{23,24}$ Izdebska et al. ${ }^{25}$ suggested in a study that long-term aerobic exercise could decrease the variability of blood pressure, improve the regulation function of the vagus nerve, improve the function of stress reflex, restore the function of the autonomic nerve, and accordingly decrease blood pressure. The results of this study revealed that t'ai chi exercise could regulate emotion in in-service subjects with hypertension and improve their quality of life. Through a 12-week study, Zheng et al. confirmed that ${ }^{26}$ t'ai chi exercise could improve the physical and mental health levels of the elderly with risk of stroke in the community. Other studies have also revealed that t'ai chi exercise could slow down stress, relieve anxiety and depression, and improve the overall physical and mental state. $^{27-29} t^{\prime}$ ai chi exercise is carried out in an aerobic environment, and can promote systemic aerobic metabolism, tune emotion, and edify disposition. Furthermore, $t$ 'ai chi is carried out in a quiet environment, which is guided by intention instead of force, and is conducive to relieving tension, improving the functions of respiration and circulation. After the exercise, participants felt relaxed, happy, and energetic, and accordingly achieve the purpose of adjusting the psychological balance and purifying the mood. Therefore, t'ai chi exercise can relieve the emotional problems of inservice subjects with hypertension and improve their ability of social adaptation. ${ }^{30}$

In summary, as a low-intensity aerobic exercise, t'ai chi exercise can improve the state of blood pressure in inservice subjects with grade 1 hypertension, improve the risk factors of cardiovascular diseases such as lipid metabolism and HR, and improve quality of life. This study has the following limitations: the subjects came from three communities; there was a lack of diversity; the follow-up duration was as short as 3 months; and the mode, time, and frequency of the t'ai chi exercise could not be closely monitored. Hence, there may be recollection deviations. In the next step, the authors should expand the sample size, prolong the follow-up duration, improve the quality of follow-ups, and stratify the subjects according to age, gender, nature, and intensity of work, to further investigate the mechanism of $t$ 'ai chi exercise in improving the risk factors of cardiovascular diseases, such as blood pressure and blood lipid metabolism, in in-service subjects with hypertension.

\section{Acknowledgments}

This study is supported by science and technology program of Traditional Chinese Medicine of Zhejiang Province: "Effect of t'ai chi on blood pressure and occupational stress in subjects with hypertension" (No. 2013ZB015); by public welfare technology application research program of Zhejiang Provincial Department: "Evaluation and application of blood pressure professional management technology for people over 18 years old based on hierarchical diagnosis and treatment" (No. 2016C33123); by medical and health science and technology program of Zhejiang Province: "Study on the correlation between blood pressure and function of target organs in normal high blood pressure group in young and middle-age" (No. 2012KYA010) and: "Study on the Elasticity in Blood Vessels in the uncontrolled essential hypertension subjects" (No. 2012KYA001); and by public welfare technology application research program of Zhejiang Provincial Department: "Study on the Curative Effects of COPD subjects Rehabilitation Exercise Prescription upon different Assessments" (No. 2015C33121).

\section{Author Disclosure Statement}

No competing financial interests exist.

\section{References}

1. National Center of Cardiovascular Diseases. Report on the Cardiovascular Disease in China, 2011 [In Chinese]. Beijing, China: Encyclopedia of China Publishing House, 2012.

2. Weber MA, Schiffrin EL, White WB, et al. Clinical practice guidelines for the management of hypertension in the community: A statement by the American Society of Hypertension and the International Society of Hypertension. J Clin Hypertens (Greenwich) 2014;16:14-26.

3. Yang L, Shou XL, Tang XH, et al. Analysis of the status of hypertension and related factors among staffs in some universities in Zhejiang province [In Chinese]. Chin J Hypertens 2015;23:52-56

4. Revision Committee of the Primary Level Management Guidelines of Hypertension in China. The primary level management guidelines of hypertension in China (revised edition, 2014) [In Chinese]. Chin J Hypertens 2015;23: 25-42.

5. Ma H, Chen ZK. Pictogram, Methods and Theories of Taichi Quan in Chen Style [In Chinese]. Beijing, China: Beijing Sport University Press, 2010:11-19.

6. Wang C, Lu W, Wu ZY. Comparison of the effects between Tai Chi and walking exercises on the rehabilitation of hypertension symptoms [In Chinese]. Mod Prevent Med 2007; 34:3535-3536.

7. Writing Group of 2010 Chinese Guidelines for the Management of Hypertension. 2010 Chinese guidelines for the management of hypertension [In Chinese]. Chin J Cardiol 2011;39:579-616.

8. Yang HX. Body-Building Prescription with Taichi Quan Among the Middle-Aged and Elderly [In Chinese]. Shanghai, China: Shanghai University of Sport, 2011.

9. Wu YX, Zhang HP. The influence of 24-pattern Taichi Quan intervention on the TXB2 and 6-K-PGF1 $\alpha$ level in the plasma among the middle-aged and aged women with hyperlipemia [In Chinese]. Chin J Gerontol 2015;35:65036505. 
10. Ware JE, Jr., Sherbourne CD. The MOS 36-item short-form health survey (SF-36). I. Conceptual framework and item selection. Med Care 1992;30:473-483.

11. Editorial Board of the Report of Cardiovascular Disease in China. Report of Cardiovascular Disease in China (2013) [In Chinese]. Beijing, China: Encyclopedia of China Publishing House, 2012.

12. Fiuza-Luces C, Garatachea N, Berger NA, et al. Exercise is the real polypill. Physiology (Bethesda) 2013;28:330-358.

13. Brook RD, Appel LJ, Rubenfire M, et al. Beyond medications and diet: Alternative approaches to lowering blood pressure: A scientific statement from the American Heart Association. Hypertension 2013;61:1360-1383.

14. Cornelissen VA, Smart NA. Exercise training for blood pressure: A systematic review and meta-analysis. J Am Heart Assoc 2013;2:e004473.

15. Lund Rasmussen C, Nielsen L, Linander Henriksen M, et al. Acute effect on ambulatory blood pressure from aerobic exercise: A randomised cross-over study among female cleaners. Eur J Appl Physiol 2018;118:331-338.

16. Dimeo F, Pagonas N, Seibert F, et al. Aerobic exercise reduces blood pressure in resistant hypertension. Hypertension 2012;60:653-658.

17. Lee YM. The effects of Tai Chi on waist circumference and blood pressure in the elderly. J Phys Ther Sci 2017;29:172175.

18. Eom AY. The effects of the Tai Chi exercise on metabolic syndrome and health-related quality of life in middle-aged women. J Muscle Joint Health 2012;19:152-160.

19. Taylor-Piliae RE. Effectiveness of tai chi exercise in improving aerobic capacity: A meta-analysis. Med Sport Sci 2008;52:40-53.

20. Motoyama M, Sunami Y, Kinoshita F, et al. The effects of long-term low intensity aerobic training and detraining on serum lipid and lipoprotein concentrations in elderly men and women. Eur J Appl Physiol Occup Physiol 1995;70: $126-131$

21. Dickie K, Micklesfield LK, Chantler S, et al. Cardiorespiratory fitness and light-intensity physical activity are independently associated with reduced cardiovascular disease risk in urban black South African women: A crosssectional study. Metab Syndr Relat Disord 2016;14:23-32.

22. Heckbert SR, Rutter CM, Oliver M, et al. Depression in relation to long-term control of glycemia, blood pressure, and lipids in subjects with diabetes. J Gen Intern Med 2010; 25:524-529.

23. Rothwell PM, Howard SC, Dolan E, et al. Effects of beta blockers and calcium-channel blockers on within-individual variability in blood pressure and risk of stroke. Lancet Neurol 2010;9:469-480.

24. Hamer M, Batty GD, Stamatakis E, et al. Hypertension awareness and psychological distress. Hypertension 2010; 56:547-550.

25. Izdebska E, Cybulska I, Izdebskir J, et al. Effects of moderate physical training on blood pressure variability and hemodynamic pattern in mildly hypertensive subjectsl. J Physiol Pharmacol 2004;55:713-724.

26. Zheng G, Xiong Z, Zheng X, et al. Subjective perceived impact of Tai Chi training on physical and mental health among community older adults at risk for ischemic stroke: A qualitative study. BMC Complement Altern Med 2017; 17:221.

27. Wang F, Lee EK, Wu T, et al. The effects of tai chi on depression, anxiety, and psychological well-being: A systematic review and meta-analysis. Int J Behav Med 2014; 21:605-617.

28. Liu X, Clark J, Siskind D, et al. A systematic review and meta-analysis of the effects of Qigong and Tai Chi for depressive symptoms. Complement Ther Med 2015;23: 516-534.

29. Sharma M, Haider T. Tai chi as an alternative and complimentary therapy for anxiety: A systematic review. J Evid Based Complement Altern Med 2015;20:143-153.

30. Wang SZ, He YM. The influence of Taichi Quan on the human physiological and mental function. J Changchun Normal Univ (Nat Sci) 2015;34:112-113.

Address correspondence to:

Xiao-Qing Jin, MD

Department of Traditional Chinese Medicine Zhejiang Hospital

Lingyin Road 12

Xihu District

Hangzhou 310013

China

E-mail: jinxq02134@163.com 